\title{
Incubation period of typhoidal salmonellosis: a systematic review and meta-analysis of outbreaks and experimental studies occurring over the last century
}

\author{
Adedoyin Awofisayo-Okuyelu ${ }^{1,2^{*}}$ (D), Noel McCarthy ${ }^{1,2,3}$, Ifunanya Mgbakor ${ }^{3,4}$ and lan Hall ${ }^{5}$
}

\begin{abstract}
Background: Salmonella Typhi is a human pathogen that causes typhoid fever. It is a major cause of morbidity and mortality in developing countries and is responsible for several outbreaks in developed countries. Studying certain parameters of the pathogen, such as the incubation period, provides a better understanding of its pathophysiology and its characteristics within a population. Outbreak investigations and human experimental studies provide an avenue to study these relevant parameters.

Methods: In this study, the authors have undertaken a systematic review of outbreak investigation reports and experimental studies, extracted reported data, tested for heterogeneity, identified subgroups of studies with limited evidence of heterogeneity between them and identified factors that may contribute to the distribution of incubation period.

Following identification of relevant studies, we extracted both raw and summary incubation data. We tested for heterogeneity by deriving the value of $\mathrm{I}^{2}$ and conducting a KS-test to compare the distribution between studies. We performed a linear regression analysis to identify the factors associated with incubation period and using the resulting $p$-values from the KS-test, we conducted a hierarchical cluster analysis to classify studies with limited evidence of heterogeneity into subgroups.
\end{abstract}

Results: We identified thirteen studies to be included in the review and extracted raw incubation period data from eleven. The value of $\mathrm{I}^{2}$ was $84 \%$ and the proportion of KS test $p$-values that were less than 0.05 was $63.6 \%$ indicating high heterogeneity not due to chance. We identified vaccine history and attack rates as factors that may be associated with incubation period, although these were not significant in the multivariable analysis ( $p$-value: 0.1). From the hierarchical clustering analysis, we classified the studies into five subgroups. The mean incubation period of the subgroups ranged from 9.7 days to 21.2 days. Outbreaks reporting cases with previous vaccination history were clustered in a single subgroup and reported the longest incubation period.

Conclusions: We identified attack rate and previous vaccination as possible associating factors, however further work involving analyses of individual patient data and developing mathematical models is needed to confirm these as well as examine additional factors that have not been included in our study.

Keywords: Incubation period, Salmonella Typhi, Systematic review, Observational studies, Experimental studies

\footnotetext{
* Correspondence: adedoyin.awofisayo-okuyelu@zoo.ox.ac.uk

${ }^{1} \mathrm{NIHR}$ Health Protection Research Unit in Gastrointestinal Infection, University of Liverpool, Liverpool, UK

2Department of Zoology, University of Oxford, Oxford, UK

Full list of author information is available at the end of the article
}

(c) The Author(s). 2018 Open Access This article is distributed under the terms of the Creative Commons Attribution 4.0 International License (http://creativecommons.org/licenses/by/4.0/), which permits unrestricted use, distribution, and reproduction in any medium, provided you give appropriate credit to the original author(s) and the source, provide a link to the Creative Commons license, and indicate if changes were made. The Creative Commons Public Domain Dedication waiver (http://creativecommons.org/publicdomain/zero/1.0/) applies to the data made available in this article, unless otherwise stated. 


\section{Background}

Typhoid fever is a systemic infection caused by the bacterium Salmonella enterica subsp. enterica serotype Typhi ( $S$. Typhi). It has been a major human pathogen for thousands of years, and while incidence has greatly reduced in developed countries, it remains a major cause of morbidity and mortality in developing countries [1]. Laboratory diagnosis of typhoid fever involves culture and identification through biochemical and serological means. Widal agglutination test was a common method until its diagnostic capabilities were questioned [2].

The mode of transmission is faecal-oral via ingestion of contaminated food and water or contact with an infected carrier [3]. Although it is a gastrointestinal infection, diarrhoea and vomiting are atypical symptoms of typhoid fever. People suffering from typhoid fever typically complain of systemic symptoms including gradual onset of fever, general malaise, headaches and abdominal pain [4].

Prevention of typhoid fever, like most other gastrointestinal infections, includes good hand hygiene [5] and exclusion of infected food handlers. In addition, typhoid vaccine has been known to offer some protection [6].

Although typhoid fever in developed countries is mostly a sporadic disease associated with travel to endemic countries, outbreaks are still commonly reported $[7,8]$. Factors such as improper cooling, inadequate heating of food, contact with contaminated raw products and infected food handlers have been implicated as contributory factors of outbreaks [9].

Investigation of outbreaks contributes to the control and reduction of the burden of disease by identifying and eliminating the source of infection. Information obtained from observational studies of outbreak investigations, particularly outbreaks with well-defined point of exposures, as well as experimental studies provide a means of studying and understanding the disease by studying certain parameters in real-life and under laboratory conditions.

Incubation period of typhoid fever, which is the time between exposure and onset of clinical symptoms, is one of the essential parameters to be studied. It is expected to be a distribution rather than a single estimate as a result of individual variation based on factors such as dose response, food matrix and host characteristics. An accurate knowledge of the incubation period distribution of typhoid fever is relevant for surveillance and implementation of public health interventions. It also contributes towards effective outbreak investigation as incorrect estimations can be misleading if they were used to determine the time of exposure [10]. Accurate knowledge of the incubation period helps in correctly classifying primary and secondary cases and exclusion of travel related cases. The incubation period also offers insights into the pathophysiology of typhoid fever and is important in conducting epidemiological and ecological studies [11].
Despite the importance of knowing the accurate incubation period distribution of typhoid fever, large organisations give a wide range with no clear indication of which durations are common and which are rare. According to the World Health Organisation (WHO), incubation periods ranging from three to sixty days have been reported [3]. The Centres for Disease Control and Prevention (CDC) reported the incubation period as three to thirty days [12].

In this study, we have systematically reviewed the literature for outbreaks of well-defined point source exposures and human experimental studies of typhoid fever with the aim of estimating the distribution of the incubation period and identify factors that may explain any variation observed. We extracted summary estimates and individual incubation period data reported, tested for the presence of heterogeneity, identified factors that may contribute to heterogeneity and defined subgroups of studies than can be combined for analysis.

\section{Methods}

Research questions

The questions we aimed to answer in this review were:

1. What is the distribution of incubation period of Salmonella Typhi in humans?

2. What factors influence the incubation period?

\section{Modified PICO elements}

Population studies /Participants - Laboratory confirmed cases of Salmonella Typhi in a point source exposure outbreak or continuous source outbreaks where date of exposure and onset is known for each case or experimental study.

Probable cases of typhoidal salmonellosis based on clinical presentation and case definitions in the context of a point source exposure outbreak.

Infectious agent - Salmonella enterica subsp. enterica serotype Typhi.

Comparator - Host factors and any other factors such as vaccine history, ingested dose, food vehicle, case definitions.

Outcome - Time from exposure to onset of clinical illness as described or defined by the authors including fever, abdominal pain, vomiting etc.

\section{Literature search}

The authors carried out a systematic literature search of peer reviewed publications on PubMed to identify observational studies and experimental studies reporting incubation period. Combining all the terms of interest, we formed the compound search string: Salmonella Typhi AND Humans AND (Outbreak* OR Experiment*). The reference list of review papers was also screened to 
identify other relevant studies that may have been missed in the original search. The search was carried out between 29 May to 24 June 2017.

\section{Selection process}

Each article went through a rigorous selection process, and relevant articles were assessed for the quality of reported data. The selection and assessment process were done in the following phases:

1) Screening of titles and abstracts for articles reporting typhoidal salmonellosis

2) Screening of full texts for reporting of incubation period

3) Review of full texts to assess exposure times and quality of reported data according to defined quality assessment criteria.

The quality assessment criteria used in this review has previously been explained in Awofisayo-Okuyelu et al. [13]. The process involved two reviewers independently assessing each study and comparing results. Where there was a difference in opinions, discussions were held until a consensus was reached.

\section{Data extraction process}

Relevant data was extracted using a pre-developed pro forma (Table 1). The type of data extracted from all studies included general information on the article, characteristics of the observational study or experimental study, details of the organism, attack rate, setting of exposure, details of case definition and summary measures of the incubation period such as mean, median, mode and range where available.

Some studies further reported individual incubation data either as an epidemic curve or descriptive table. Where an epidemic curve was provided, we used a free online data extraction tool called WebPlotDigitizer version 3.10 [14] to extract the individual incubation data. If data was provided as a descriptive summary table, individual incubation data was equally extracted.

The unit of measurement reported and extracted was in days.

\section{Descriptive analyses}

We calculated frequencies and percentages, summarising the characteristics of all studies. Characteristics summarised included: study design (observational or experimental), study type for observational studies (case-control, cohort or descriptive), year of study, country of study, age distribution of cases, mode of transmission and setting of exposure.

Using the extracted individual incubation data, we re-created the epidemic curves of the studies plotting each graph on a standard $x$-axis indicating incubation period ranging from zero to forty-five days and individual $y$-axis indicating number of cases in the outbreak or experimental study.

Table 1 Details of data extracted

\begin{tabular}{|c|c|}
\hline Section & Information to be collected \\
\hline General information & $\begin{array}{l}\text { - Year of publication } \\
\text { - Title of article } \\
\text { - Authors } \\
\text { - Type of publication (journals, conference abstract, grey literature, etc.) } \\
\text { - PubMed ID (where applicable) }\end{array}$ \\
\hline Study characteristics & $\begin{array}{l}\text { - Year of study } \\
\text { - Study design (cohort, case-control, experimental, case series) } \\
\text { - Country of study } \\
\text { - Age distribution } \\
\text { - Comments on method or quality of study }\end{array}$ \\
\hline Pathogen characteristics & $\begin{array}{l}\text { - Infectious agent } \\
\text { - Species } \\
\text { - Subtype }\end{array}$ \\
\hline Outcome data/ results & $\begin{array}{l}\text { - Case definition } \\
\text { - Reported incubation period (individual data, mean, median mode and range) } \\
\text { - Derived or calculated summary estimates incubation period (raw data extracted) } \\
\text { - Source of calculated data (epidemic curve or author description) }\end{array}$ \\
\hline Other outcome data & - Incubation period to particular symptoms \\
\hline Factors that could affect incubation period & $\begin{array}{l}\text { - No of exposed cases } \\
\text { - No of people affected } \\
\text { - Setting } \\
\text { - Mode of transmission } \\
\text { - Food vehicle (for foodborne infections only) } \\
\text { - Patient characteristics (e.g. age, previous infection or treatment, underlying illness) }\end{array}$ \\
\hline Any other relevant information & - Any other relevant information \\
\hline
\end{tabular}




\section{Statistical analyses}

Using the individual incubation data, we tested for the presence of heterogeneity and defined the pattern of heterogeneity. Using both the individual and summary incubation data, we identified factors that may explain heterogeneity. Analyses were carried out using the statistical software R version 3.2.3 (2015-12-10) - "Wooden Christmas Tree" [15]. Further details on the statistical analyses is described in Awofisayo-Okuyelu et al. [13].

\section{Testing for heterogeneity}

We tested for heterogeneity across the studies in two ways. First, we calculated the value of $\mathrm{I}^{2}$ by deriving the Q statistic and inputting it into the formula: $I^{2}=100 \%$ $X(Q-d f) / Q$; where $\mathrm{Q}$ is the Cochran's heterogeneity statistic and $d f$ is the corresponding degree of freedom. A $p$-value of less than 0.05 from the $\mathrm{Q}$ statistic test provided evidence of heterogeneity between the studies. Furthermore, the value of $\mathrm{I}^{2}$ was interpreted according to the Cochran suggested threshold [16] to determine the magnitude of heterogeneity.

The second way we tested for heterogeneity was by performing a two-sample Kolmogorov-Smirnov test (KS test) which compares the cumulative distributions between the studies. We applied a bootstrapped version of the function, repeating the sampling 100,000 times in order to derive $p$-values that will provide improved coverage due to potential ties in the data comparisons.

The output of the KS test was the D-statistic and the corresponding $p$-value. A high $\mathrm{d}$-statistic value and a low $p$-value indicated the presence of heterogeneity. We further compared the $p$-values to confirm if any observed heterogeneity was due to chance. We calculated the proportion of $p$-values below 0.05 and the probability of obtaining the observed proportion. Statistical evidence of heterogeneity in the reported incubation period was available if the probability was less than 0.01 .

\section{Identifying factors that explain heterogeneity}

We performed a linear regression analysis using the summary incubation data available from all studies. We fitted a generalised linear model with gamma as the family function to account for skewness of the data and the link function used was 'identity'. The effect of the explanatory variables on the mean incubation period was examined using a univariate model, and where there was a significant association ( $p$-value 0.05$)$, the associated variables were included in a multivariable model to test for confounding.

\section{Identifying subgroups of studies/describing pattern of heterogeneity}

Once heterogeneity was confirmed, the next step was to identify subgroups of studies with limited evidence of heterogeneity. We used hierarchical clustering analysis to statistically identify subgroups of studies. Studies that reported only summary data (studies 7 and 11) or aggregated raw data (studies 1 and 3) were excluded from the cluster analysis.

The $p$-value of the KS test was converted to a dissimilarity matrix and used to create a hierarchical cluster to show a graphical representation of the dissimilarities between the studies. The cluster analysis algorithm used was the

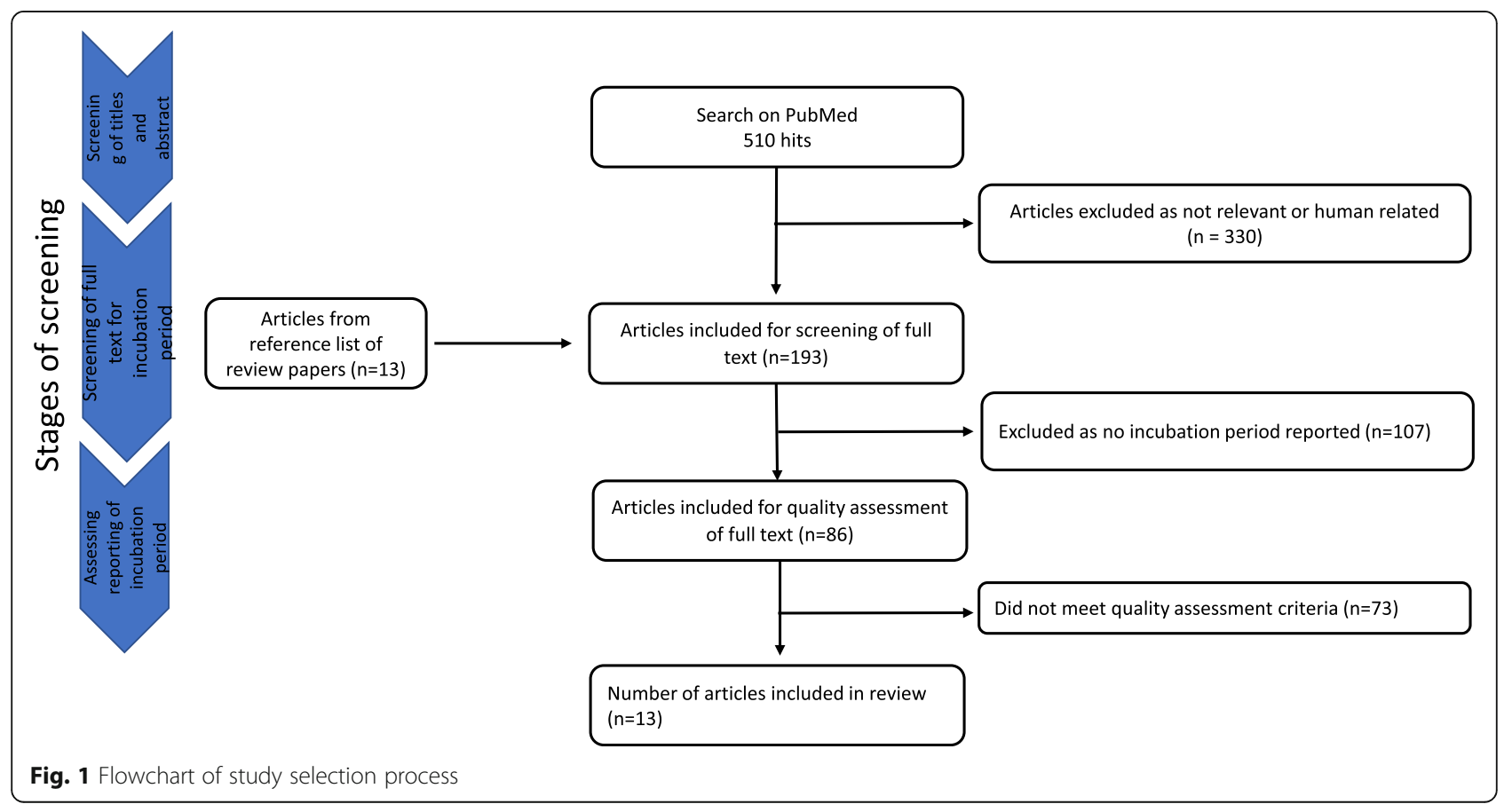


complete linkage method. The output was a dendrogram showing a compact visualisation of the dissimilarity matrix.

In order to identify clusters within the dendrogram, we had to determine a cut-off point at which the studies were significantly allocated to subgroups. Considering that the KS test was a pairwise test, thereby increasing the likelihood of a type 1 error (observing one significant result due to chance), we applied a pragmatic adjustment to the significance level (0.05) by dividing it by the number of studies included in the KS test. We then subtracted the corrected $p$-value from one to derive a cut-off point from which studies with limited evidence of heterogeneity can be defined within subgroups.

\section{Subgroup analysis}

Individual incubation period data was pooled to generate a dataset for each subgroup. Summary statistics and outcome measures were derived including:

- Number of studies included in a subgroup

- Total number of cases (sum of cases in all studies included in a subgroup)

- Mean and median incubation period of cases within a subgroup

- Variance, skew and kurtosis of incubation period of cases within a subgroup

- Mean attack rate of studies within a subgroup

- Reported vaccination history of studies within a subgroup

A forest plot showing the distribution of the mean incubation period and the corresponding 95\% confidence interval was created. The reported mean incubation period was used to allocate studies without individual patient data to appropriate subgroups for illustration.

\section{Risk of bias}

We analysed our data for the presence of small-study effect using a funnel plot to visualise the relationship between sample size and incubation period.

\section{Results}

\section{Literature search and selection process}

A total of 510 articles were retrieved following the search in PubMed. Titles and abstracts were screened to identify relevant articles. Articles were excluded if there were non-human related studies, or non-typhi studies. Studies that were not reported in English were also excluded. This resulted in 180 articles available for full text screening of incubation period data after excluding 330 irrelevant articles. Searching through the reference list of other review papers, we identified thirteen articles that were not included in the original search, bringing the number of articles available for full text screening to 193. Excluding articles that did not report incubation period resulted in 86 articles that went through the quality assessment process. Seventy-three articles did not meet the quality assessment criteria (Additional file 1) and were further excluded (Fig. 1). The resulting number of articles available for inclusion in the study was thirteen (Additional file 2), all of which reported individual incubation data with the exception of studies 7 and 11.

\section{Descriptive analysis of studies}

All studies included in our review reported incubation period of cases associated with $S$. Typhi, however, very few studies reported the microbiological characteristics (phage types) of the infecting organism.

Table 2 Characteristics of included studies

\begin{tabular}{|c|c|c|}
\hline & $\mathrm{N}$ & $\%$ \\
\hline Total number of studies & 13 & \\
\hline \multicolumn{3}{|l|}{ Year of study } \\
\hline Before year 2000 & 8 & 61.5 \\
\hline 2000 and later & 4 & 30.8 \\
\hline unknown & 1 & 7.7 \\
\hline \multicolumn{3}{|l|}{ Region of study } \\
\hline Europe & 3 & 23.1 \\
\hline North America & 5 & 38.5 \\
\hline Africa & 2 & 15.4 \\
\hline Asia & 2 & 15.4 \\
\hline Unknown & 1 & 7.7 \\
\hline \multicolumn{3}{|l|}{ Study design } \\
\hline Descriptive & 4 & 30.8 \\
\hline Case control & 4 & 30.8 \\
\hline Retrospective cohort & 3 & 23.1 \\
\hline Experimental study & 2 & 15.4 \\
\hline \multicolumn{3}{|l|}{ Setting of exposure } \\
\hline Catered meal & 5 & 38.5 \\
\hline Outdoor activity & 2 & 15.4 \\
\hline Picnic & 2 & 15.4 \\
\hline Experimental study & 2 & 15.4 \\
\hline Community & 1 & 7.7 \\
\hline Restaurants & 1 & 7.7 \\
\hline \multicolumn{3}{|l|}{ Food vehicle category } \\
\hline Red meat & 2 & 15.4 \\
\hline Dairy and dairy products & 2 & 15.4 \\
\hline Salad & 3 & 23.1 \\
\hline Non-foodborne & 1 & 7.7 \\
\hline Other & 3 & 23.1 \\
\hline Unknown & 2 & 15.4 \\
\hline
\end{tabular}


Studies included in our review were published between 1914 and 2016. Sixty one percent (8/13) of the outbreaks or experiments took place before year 2000 with the majority $(5 / 13 ; 38.5 \%)$ occurring the North America (Table 2). Two studies were experimental and the remainder were epidemiological studies including descriptive studies (4/ $13 ; 30.8 \%)$, case control studies $(4 / 13 ; 30.8 \%)$ and retrospective cohort studies $(3 / 13 ; 23.1 \%)$. The eleven outbreaks included resulted in a total of 635 cases.

The settings of exposure varied and the most common reported setting was events with catered meals (5/13; $38.5 \%)$. Outdoor activities and picnic events were reported in two outbreaks each $(2 / 13 ; 15.4 \%)$. With the exception of one outbreak that was caused by exposure to surface water at a recreational park, all infections were foodborne and the most frequently reported food vehicles were salad and vegetables $(3 / 13 ; 23.1 \%)$, red meat and dairy both accounting for two outbreaks each $(2 / 13 ; 15.4 \%)$. The food vehicle for two outbreaks was unknown. (Table 2).

The funnel plot did not indicate any effect of study size on the reported incubation period as it was symmetric and the data points were evenly distributed around the $\mathrm{x}$-axis (Additional file 3 ).
Reviewing the re-created epidemic curves from the eleven studies that provided individual incubation data, we observed a range of distributions in the plots (Fig. 2). The mean incubation period ranged from 7 days to 21.4 days. The minimum incubation period reported was 2 days and the maximum was 41 days (Table 3 ).

Identification of cases was dependent on the case definition given by the authors of each study. In eight studies, microbiological confirmation was used to define cases, in two studies (studies 6 and 13), a very specific case definition including epidemiological link was used and in three studies, the case definition was less specific but also including epidemiological link (Table 3).

Of the twelve studies where the attack rates could be calculated, five had an attack rate higher than 50\% and the highest attack rate was $79.31 \%$ in an outbreak involving ingestion of contaminated ice cream [17]. This outbreak also recorded the second highest case fatality rate.

Five studies reported vaccination history of cases, and of these, two reported previous vaccination of some or all of their cases. These two studies reported the longest mean incubation period of 21.4 and 20.9 days. The attack rates

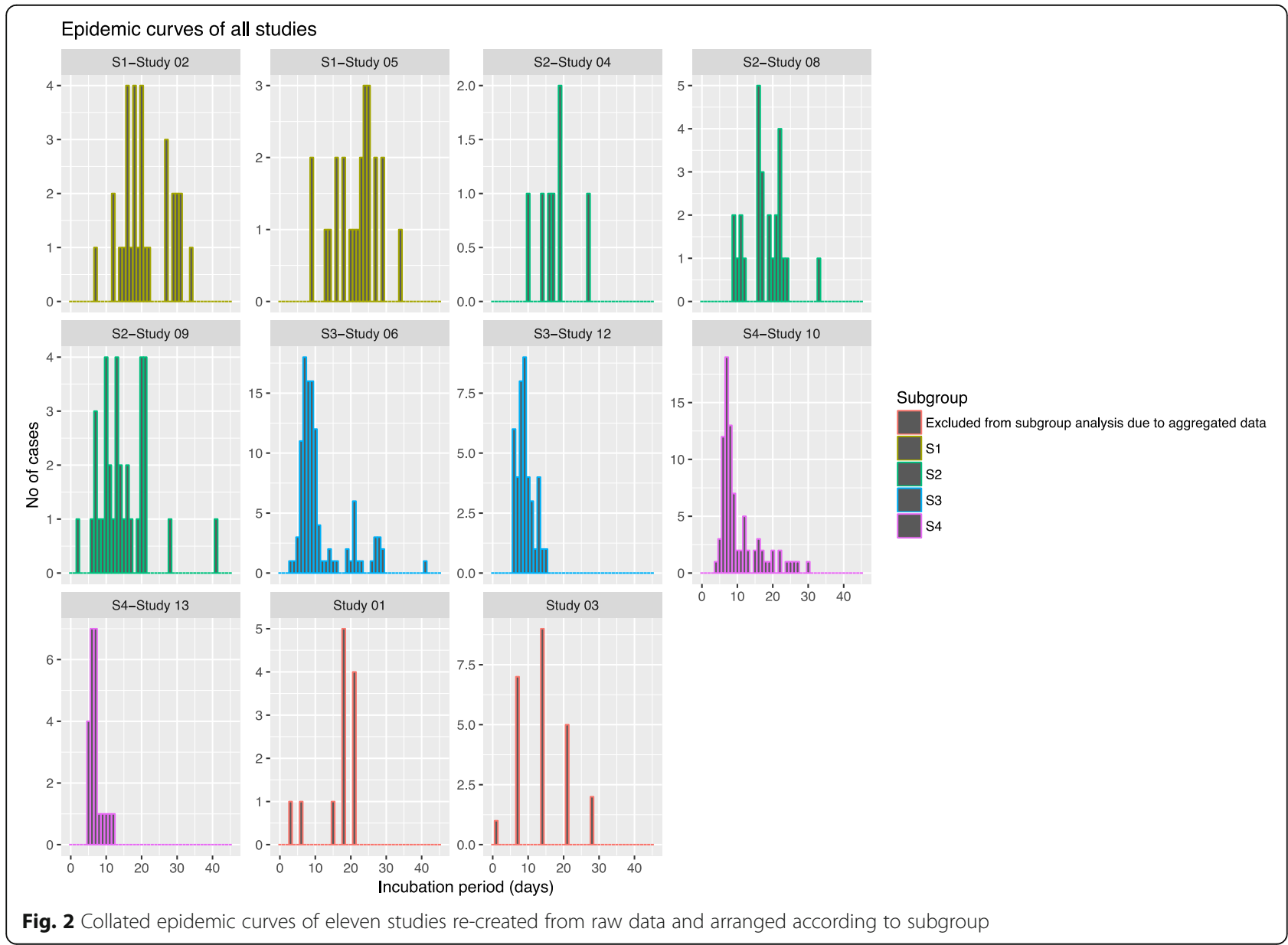




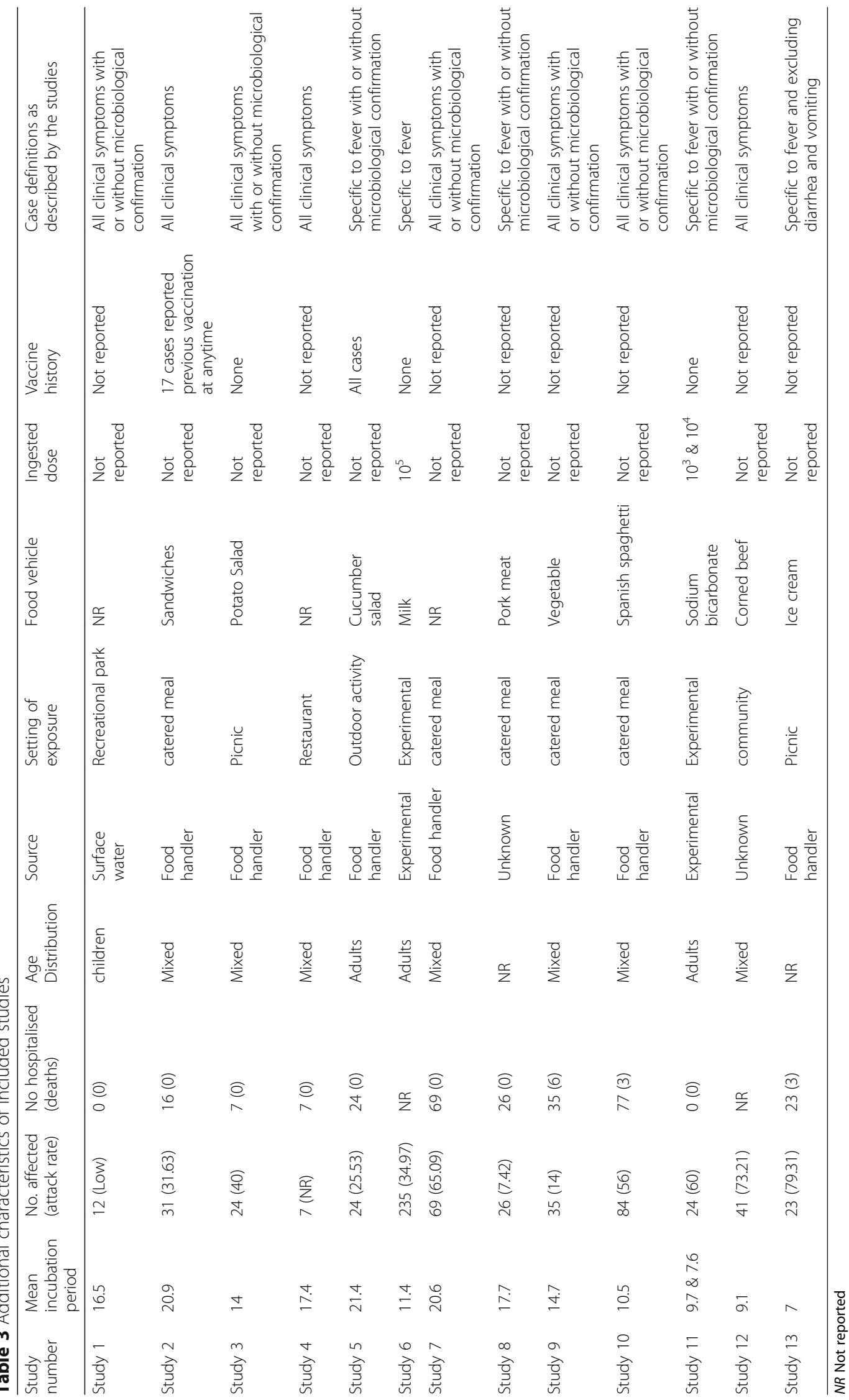


in these populations were below 50\%, however, these were not the lowest attack rates reported.

\section{Test for heterogeneity}

The $p$-value of the derived Q statistic was $<0.0001$ and the calculated value of $\mathrm{I}^{2}$ was $84 \%$ indicating high heterogeneity in the reported incubation period across the studies. The proportion of KS test $p$-values that were less than 0.05 was $63.6 \%(42 / 66)$ and the probability of obtaining the observed proportion was $<0.0001$ further confirming the high heterogeneity across the studies which is not due to chance.

\section{Factors that may explain heterogeneity}

Results of the univariate analysis showed that previous vaccination history, attack rate and year of study had a significant association with the mean incubation period. The mean incubation period reduced by 1.4 days with every $10 \%$ increase in attack rate ( $p$-value 0.05$)$, and outbreaks containing cases that had been vaccinated reported a longer incubation period of 9.8 days compared to outbreaks without vaccinated cases (Table 4). Outbreaks that occurred after 1950 reported a longer incubation period of 6.8 days compared to outbreaks that occurred before 1950. When these variables were included in the multivariable analysis, there was no significant association between vaccination, year of study and mean incubation. The effect observed for year of study was reversed such that outbreaks that occurred after 1950 now reported a shorter incubation period of 3.9 days.

\section{Identifying subgroups of studies}

Based on the clustering analysis, studies were paired according to the evidence of dissimilarities between them. Studies found to have the least evidence of dissimilarities were paired and then connected by branches to another pair of studies or a single study with minimal dissimilarity

Table 4 Linear regression analysis to identify factors associated with the distribution of incubation period

\begin{tabular}{|c|c|c|c|c|}
\hline \multirow[t]{2}{*}{ Variables } & \multicolumn{2}{|l|}{ Univariate analysis } & \multicolumn{2}{|l|}{ Multivariable analysis } \\
\hline & Difference in mean & $p$-value & Difference in mean & $p$-value \\
\hline \multicolumn{5}{|l|}{ Age distribution } \\
\hline Adult & Reference & & & \\
\hline Children & 2.7 & 0.6 & & \\
\hline Mixed ages & 1.5 & 0.6 & & \\
\hline \multicolumn{5}{|l|}{ Year of study } \\
\hline Pre 1950 & Reference & & & \\
\hline Post 1950 & 6.8 & 0.01 & -3.9 & 0.1 \\
\hline Attack rate & -0.14 & 0.05 & -0.26 & 0.07 \\
\hline \multicolumn{5}{|l|}{ Setting } \\
\hline Restaurant & Reference & & & \\
\hline Catered meal & -0.5 & 0.9 & & \\
\hline Community & -8.3 & 0.1 & & \\
\hline Experimental & -7.4 & 0.2 & & \\
\hline Outdoor activity & 1.5 & 0.8 & & \\
\hline Picnic & -6.9 & 0.2 & & \\
\hline \multicolumn{5}{|l|}{ Food category } \\
\hline Other & Reference & & & \\
\hline Dairy & -4.3 & 0.3 & & \\
\hline Non-food & 3.1 & 0.6 & & \\
\hline Red meat & 0.1 & 0.9 & & \\
\hline Salad \& vegetable & 3.3 & 0.5 & & \\
\hline \multicolumn{5}{|l|}{ Vaccine history } \\
\hline None & Reference & & & \\
\hline Yes & 10.3 & 0.03 & 4.2 & 0.1 \\
\hline \multicolumn{5}{|l|}{ Specific case definition } \\
\hline No & Reference & & & \\
\hline Yes & -2.2 & 0.4 & & \\
\hline
\end{tabular}


to them. These pairing and connections of studies create a dendrogram of the dissimilarity matrix (Fig. 3).

The pragmatic adjustments made to the significance level resulted in a corrected $p$-value of 0.004 and a corresponding cut-off point of 0.994 . At this cut-off point, after taking into account multiple testing, four subgroups were identified. These included: one subgroup of three studies and three subgroups of two studies each (Fig. 3).

\section{Summary of subgroup analysis}

The mean incubation period was different between the subgroups (Fig. 4) and significantly decreased from subgroup 1 to subgroup 3. Subgroup 4 had the shortest mean incubation period of 9.7 days $(95 \% \mathrm{CI}$ 8.6-10.7) and subgroup 1 had the longest mean of 21.2 days (95\% CI 19.5-22.9) (Table 5). We also observed some differences in the variance, skew and kurtosis between subgroups. Subgroups 3 and 4 had the highest mean attack rate and also had the shortest incubation periods. Cases in subgroup 1 reported previous vaccination and also reported the longest incubation period.

\section{Discussion}

We have undertaken a systematic review to describe the distribution of incubation period of Salmonella Typhi. We tested for the presence of heterogeneity, attempted to identify factors that could influence the incubation period and classified the studies into subgroups with limited evidence of heterogeneity. Due to the high heterogeneity amongst the studies, estimating a single distribution of incubation period was impossible. However, we defined subgroups of similar studies and found their mean incubation period to range from 9.7 days to 21.2 days. These values are within the range quoted by international organisations reporting 3 to 60 days [3], 3 to 30 days [12] and also scientific literatures reporting 10 to 20 days [18]. Some literature report shorter incubation periods than we observed, like Raffatellu et al. reporting a median of 5 to 9 days [19] or 8 to 14 days [18]. We also identified attack rate and vaccination as possible factors that could affect the incubation period distribution.

The relationship between attack rate and incubation period is inversely proportional, such that higher attack rates result in shorter incubation periods $[20,21]$. This has been reported in numerous outbreaks [22-24] and also observed in our review. Attack rates are associated with factors such as virulence of the organism, host characteristics and infecting dose [22]. When either of these factors are present in a way that increases the attack rate: high virulence, susceptible host and large infecting dose, the incubation period is shortened as the onset of illness is quicker.

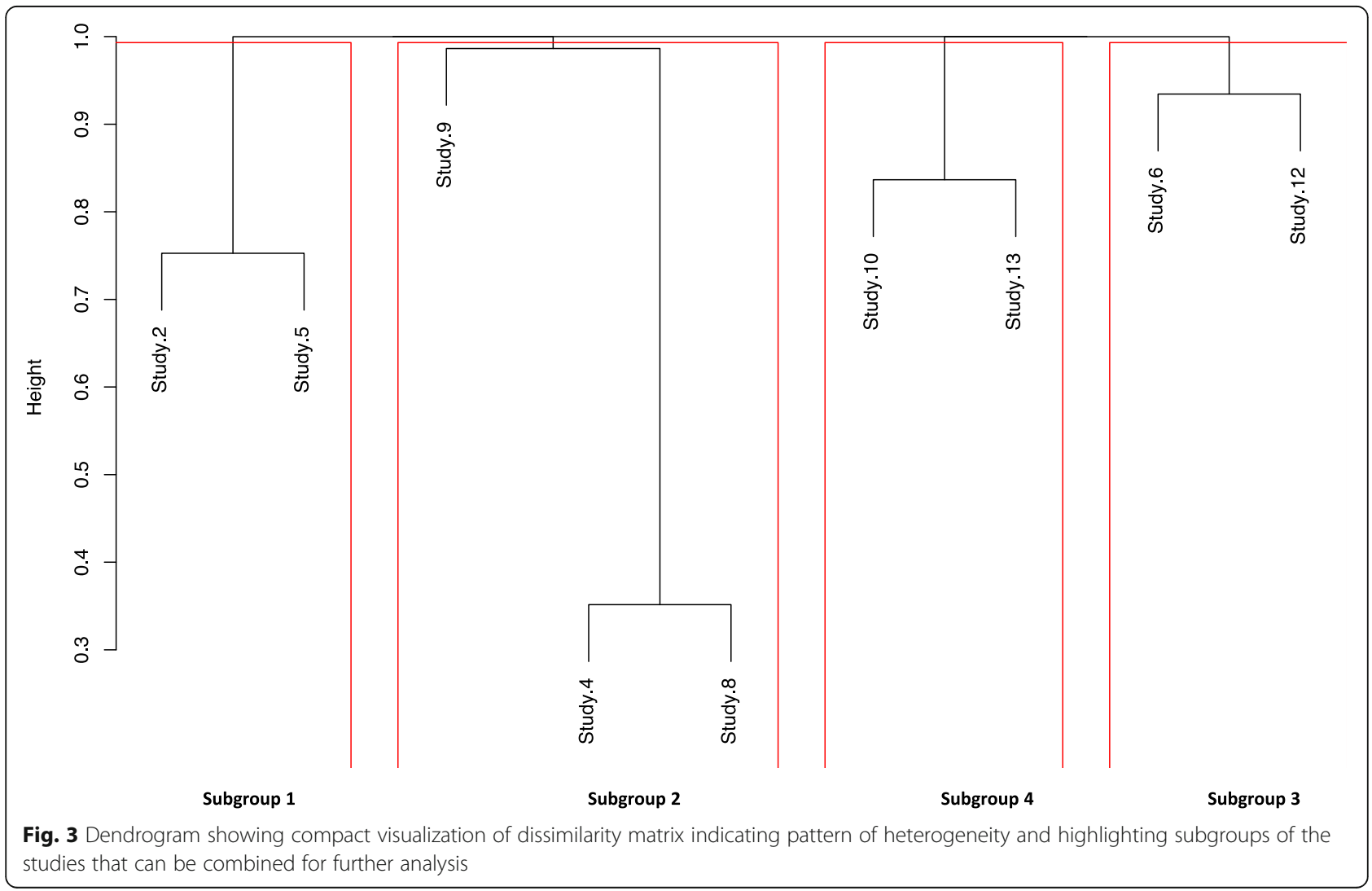




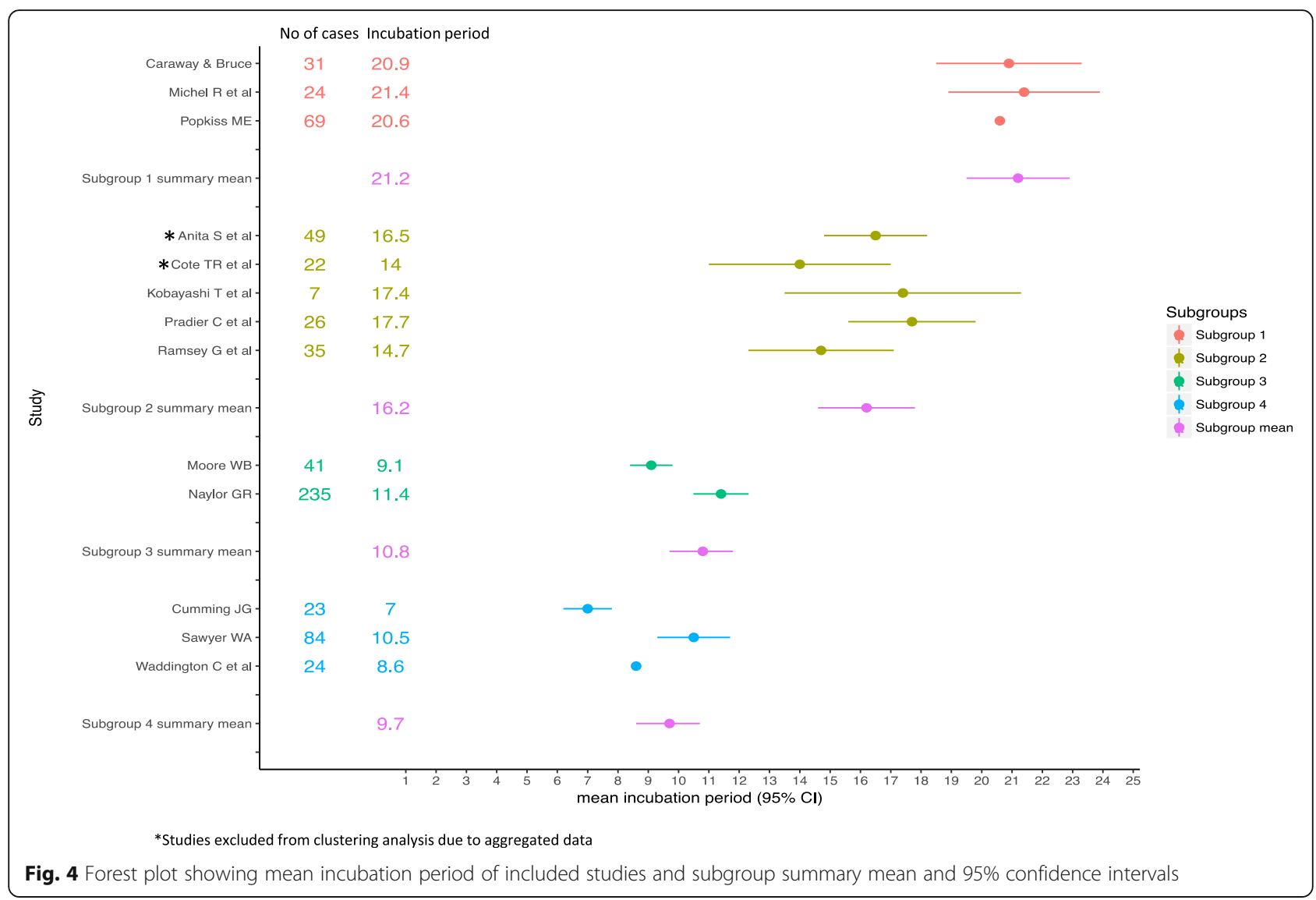

Vaccination is one of the preventive measures advised by public health professionals $[25,26]$ to control or mitigate the burden of disease brought on by $S$. Typhi [27]. It is recommended to people living in or visiting endemic areas and to control outbreaks [3]. Our study did not aim to review the efficacy of typhoid vaccine in preventing outbreaks, however we identified a couple of studies with outbreaks reported in vaccinated population $[28,29]$ and similar studies have also been reported [30]. As observed from our review, we may say that in populations where vaccination does not prevent illness, it may delay the onset of symptoms thereby prolonging the incubation period. This could have an adverse effect in outbreaks with continuous source as more people will be exposed and possibly infected before the outbreak is discovered.
The subgroup analysis indicated that outbreaks and experimental studies that took place before the year 1950 belonged to subgroups reporting shorter incubation period (subgroups 3 and 4). In the univariate analysis, post-1950 studies had a significantly longer incubation period, however, in the multivariate analysis, this effect on the incubation period was reversed as we observed a shorter incubation period in post-1950 studies. The change in the direction of effect is indicative of the presence of a confounding factor in the univariate analysis.

A lot of reported outbreaks caused by $S$. Typhi either had a continuous source of exposure such as municipal water supply [31-34], or were caused by widely distributed food produce $[35,36]$. This means that point

Table 5 Summary statistics of outbreaks and incubation period within subgroups

\begin{tabular}{llllllllll}
\hline & Count & Sum of cases & Median & Mean $(95 \% \mathrm{Cl})$ & Variance & Skew & Kurtosis & Attack rate & Vaccine \\
\hline Subgroup 1 & 2 & 55 & 20 & $21.2(19.5-22.9)$ & 42.2 & -0.002 & -0.6 & 28.7 & All or most cases \\
Subgroup 2 & 3 & 68 & 16 & $16.2(14.6-17.8)$ & 42.7 & 0.9 & 2.3 & NR & Unknown \\
Subgroup 3 & 2 & 276 & 9 & $10.8(9.7-11.8)$ & 39.7 & 2.1 & 4.4 & 54.1 & None \\
Subgroup 4 & 2 & 107 & 8 & $9.7(8.6-10.7)$ & 29.3 & 1.8 & 2.9 & 67.6 & Unknown \\
\hline
\end{tabular}


source outbreaks, where the time of exposure is definite and incubation period could be accurately calculated, were few. This was a similar finding in the review conducted by Naylor [24]. The author stated the criteria of an outbreak suitable for incubation period analysis and reported that very few reports fulfilled these criteria. However, in their review, studies where the date of exposure was not confirmed were included and date of infection was inferred from date of purchase, date of sale for a batch of food item, date of water contamination and so on. Continuous source outbreaks can be useful if the investigators identify and report the exact dates of exposure and onset but this was not the case in the outbreaks reviewed by Naylor. Hence, some studies identified in Naylor's review were not included in our study. In our study, we identified only 13 studies with point source exposures or where the time of exposure was known. Some studies however reported point source exposures, although the exposures could have occurred over a few days $[37,38]$.

Dose response is known to be associated with incubation period [20,39], and although this was not particularly studied in our review, there were studies were this relationship was observed. In an outbreak associated with ice cream [17], a dose response relationship was observed as those who had a mixture of ice creams had longer incubation periods while those who had only the contaminated ice cream had comparatively shorter incubation periods. Another dose response relationship was observed in the experimental study conducted by Waddington et al. [21]. Two cohorts of volunteers were infected with different doses of inoculum, $10^{3}$ and $10^{4}$. The cohort receiving the lower dose had a longer incubation period compared to those receiving the higher dose.

The case definition used in identifying cases or in including cases as part of an outbreak is very subjective and based on what the authors identify as a case. This is quite essential because it can either prolong or shorten the incubation period of a case based on what is considered as the onset of symptoms. Although there are guidelines on what to consider when defining a case [40], case definitions depend on the authors' perception. The complex syndrome of typhoid fever [7] makes the process of defining cases arbitrary. In our review, some of the case definitions were broad and syndromic, such as 'clinical illness', while others are very specific in terms of fever at a particular temperature $[21,29,41]$ or dismissing symptoms such as diarrhoea and vomiting [17]. Neither the broad nor specific definitions are incorrect, however, the varied case definitions may have contributed to the observed heterogeneity.

A limitation we encountered in this review was the difference in the reported incubation period data extracted from the included studies. Although the raw incubation times were reported in days, some studies reported an aggregated date of symptom onset in three or seven-day intervals $[42,43]$. Onset times were either rounded up or rounded down to fall into the reported interval. Rounding down the onset time from three days to one will spuriously shorten the incubation period just as rounding up the onset time from one day to seven will spuriously prolong the incubation period. We therefore excluded these studies from the hierarchical cluster analysis and their reported mean incubation periods should be interpreted with caution.

\section{Conclusions}

Our study showed that the reported incubation period varied more than could be explained by chance and identified attack rate and previous vaccination as associated factors, although not significantly. This may be due to the limited information available from the small number of studies we reviewed. Analysing individual patient data will provide an opportunity to assess additional patient characteristics such as underlying medical conditions, drug interactions and full vaccination history. In addition, using mathematical models to describe the process of infection will help identify parameters intrinsic to the infection pathway that could influence the distribution of incubation period such as gastric transit times, phagocyte characteristics and cellular bacteria growth.

In order to maximise the use of outbreak reports in studying parameters such as incubation period, authors should endeavour to precisely report exposure times and onset times. Where the outbreak is non-point source, identifying and reporting the exposure time of each case where possible will be useful. Case definitions should be developed in such a way as to ensure that cases are included based on similar thresholds which can also be comparable with other outbreak reports.

\section{Additional files}

Additional file 1: List of studies excluded from the review. Table listing the studies excluded from the review. (DOCX $24 \mathrm{~kb}$ )

Additional file 2: List of included studies and data. Spreadsheet containing a list of studies included in the review and the data extracted from the studies. (XLSX $14 \mathrm{~kb}$ )

Additional file 3: Funnel plot. Graph of funnel plot showing the effect of study size on the incubation period. (PDF $5 \mathrm{~kb}$ )

Additional file 4: PRISMA 2009 checklist. Table of PRISMA checklist. (DOC $73 \mathrm{~kb}$ )

\section{Abbreviations}

KS test: Kolmogorov-Smirnov test; NR: Not reported

\section{Funding}

The research was funded by the National Institute for Health Research Health Protection Research Unit in Gastrointestinal Infections at University of Liverpool in partnership with Public Health England, in collaboration with University of East Anglia, University of Oxford and the Institute of Food Research. Ian Hall is partly funded by the National Institute for Health 
Research Health Protection Research Unit in Emergency Preparedness and Response at King's College London and in Modelling Methodology at Imperial, both in partnership with Public Health England, he is also a Member of National Institute for Health Research Health Protection Research Units in Emerging and Zoonotic Infections and Gastrointestinal Infections at Liverpool. Adedoyin Awofisayo-Okuyelu is based at University of Oxford. The views expressed are those of the author(s) and not necessarily those of the National Health Service, the National Institute for Health Research, the Department of Health or Public Health England.

\section{Availability of data and materials}

The raw data on which the conclusions of this study are based have been provided as an additional file (Additional file 2) as part of this manuscript. A PRISMA checklist is also available as an additional file (Additional file 4).

\section{Disclaimer}

The views expressed are those of the author(s) and not necessarily those of the NHS, the NIHR, the Department of Health, Public Health England.

\section{Authors' contributions}

AA-O collated the data, designed the study, screened the articles as the main reviewer, conducted the data analysis and interpretation and drafted the manuscript. NM participated in the study design and critically reviewed the manuscript. IM participated in screening the articles as the second reviewer and reviewed the manuscript. IH participated in the data analyses and critically reviewed the manuscript. All authors have read and approved the manuscript.

\section{Author's information}

Adedoyin is currently a DPhil student at the Department of Zoology, University of Oxford. Her research focuses on understanding and describing the distribution of incubation period of common gastrointestinal pathogens.

\section{Ethics approval and consent to participate}

Not applicable

\section{Consent for publication}

Not applicable

\section{Competing interests}

The authors declare that they have no competing interests.

\section{Publisher's Note}

Springer Nature remains neutral with regard to jurisdictional claims in published maps and institutional affiliations.

\section{Author details}

${ }^{1} \mathrm{NIHR}$ Health Protection Research Unit in Gastrointestinal Infection, University of Liverpool, Liverpool, UK. ${ }^{2}$ Department of Zoology, University of Oxford, Oxford, UK. ${ }^{3}$ Warwick Medical School, University of Warwick, Warwick, UK. ${ }^{4}$ Epidemiology, Strategic Information and Health Systems Strengthening Branch, Nigeria Office, Lagos, Nigeria. ${ }^{5}$ School of Mathematics, University of Manchester, Manchester, UK

Received: 29 January 2018 Accepted: 17 September 2018 Published online: 27 September 2018

\section{References}

1. Pang T. Typhoid fever research in developing countries. J Infect Dev Ctries. 2008;2(6):411.

2. Tsang R, Wong A, Li K. Identification and serotyping of Salmonella typhi. Lab Med 1989;20(11):767-71.

3. World Health Organization. Background document: The diagnosis, treatment and prevention of typhoid fever [Internet]. [cited 2017 Oct 10]. Report No.: WHO/N\&B/03.07. Available from: http://www.who.int/rpc/ TFGuideWHO.pdf

4. Dougan G, Baker S. Salmonella enterica serovar Typhi and the pathogenesis of typhoid fever. Annu Rev Microbiol. 2014;68:317-36.

5. Greenwell J, McCool J, Kool J, Salusalu M. Typhoid fever: hurdles to adequate hand washing for disease prevention among the population of a peri-urban informal settlement in Fiji. West Pac Surveill Response J WPSAR. 2013:4(1):41-5.

6. Jin C, Gibani MM, Moore M, Juel HB, Jones E, Meiring J, et al. Efficacy and immunogenicity of a Vi-tetanus toxoid conjugate vaccine in the prevention of typhoid fever using a controlled human infection model of Salmonella Typhi: a randomised controlled, phase 2b trial. Lancet. 2017 0(0). Available from: http:// www.thelancet.com/journals/lancet/article/PIIS0140-6736(17)32149-9/abstract. [cited 2017 Oct 10].

7. Parry CM, Hien TT, Dougan G, White NJ, Farrar JJ. Typhoid fever. N Engl J Med. 2002;347(22):1770-82

8. Mogasale V, Maskery B, Ochiai RL, Lee JS, Mogasale W, Ramani E, et al. Burden of typhoid fever in low-income and middle-income countries: a systematic, literature-based update with risk-factor adjustment. Lancet Glob Health. 2014;2(10):e570-80.

9. Bryan FL. Risks of practices, procedures and processes that lead to outbreaks of foodborne diseases. J Food Prot. 1988;51(8):663-73.

10. Sawyer WA. Ninety-three persons infected by a typhoid carrier at a public dinner. J Am Med Assoc. 1914;LXIII(18):1537-42.

11. Nishiura H, Mizumoto K, Ejima K, Zhong Y, Cowling BJ, Omori R. Incubation period as part of the case definition of severe respiratory illness caused by a novel coronavirus. Euro Surveill Eur Commun Dis Bull. 2012;17(42). Available from: http://www.ncbi.n/m.nih.gov/pmc/articles/PMC3722063/. [cited 2016 Dec 21].

12. Centres for Disease Control and Prevention. Chapter 3: Infectious diseases related to travel - Typhoid \& Paratyphoid fever. In: CDC Yellow Book 2018 [Internet]. Oxford University Press; 2017 [cited 2017 Oct 10]. Available from: https://wwwnc.cdc.gov/travel/yellowbook/2018/infectious-diseases-relatedto-travel/typhoid-paratyphoid-fever

13. Awofisayo-Okuyelu A, Hall I, Adak G, Hawker II, Abbott S, McCarthy N. A systematic review and meta-analysis on the incubation period of campylobacteriosis. Epidemiol Infect. 2017;145(11):2241-53.

14. WebPlotDigitizer - Copyright 2010-2016 Ankit Rohatgi [Internet]. [cited 2016 Jul 19]. Available from: https://automeris.io/WebPlotDigitizer/

15. R: A language and environment for statistical computing. $R$ foundation for statistical computing. [Internet]. Vienna; 2015. Available from: https: //https:// www.r-project.org

16. Higgins J, Green S (editors). Cochrane handbook for systematic reviews of interventions Version 5.1.0 [updated March 2011]. Cochrane Collab [Internet]. 2011 [cited 2016 Apr 22]; Available from: www.handbook.cochrane.org

17. Cumming JG. An epidemic resulting from the contamination of ice cream by a typhoid carrier. J Am Med Assoc. 1917;LXVIII(16):1163-5.

18. Freedman J, Lighton $L$, Jones J. Defining travel-associated cases of enteric fever. J Infect Public Health. 2014;7(5):377-85.

19. Raffatellu M, Wilson RP, Winter SE, Bäumler AJ. Clinical pathogenesis of typhoid fever. J Infect Dev Ctries. 2008;2(4):260-6.

20. Glynn JR, Hornick RB, Levine MM, Bradley DJ. Infecting dose and severity of typhoid: analysis of volunteer data and examination of the influence of the definition of illness used. Epidemiol Infect. 1995;115(1):23-30.

21. Waddington CS, Darton TC, Jones C, Haworth K, Peters A, John T, et al. An outpatient, ambulant-design, controlled human infection model using escalating doses of Salmonella typhi challenge delivered in sodium bicarbonate solution. Clin Infect Dis Off Publ Infect Dis Soc Am. 2014;58(9): $1230-40$.

22. Glynn JR, Bradley DJ. The relationship between infecting dose and severity of disease in reported outbreaks of Salmonella infections. Epidemiol Infect. 1992;109(3):371-88.

23. Blaser MJ, Newman LS. A review of human salmonellosis: I. infective dose. Rev Infect Dis. 1982;4(6):1096-106.

24. Naylor GR. Incubation period and other features of food-borne and waterborne outbreaks of typhoid fever in relation to pathogenesis and genetics of resistance. Lancet Lond Engl. 1983;1 (8329):864-6.

25. Choices NHS. Typhoid fever - Vaccination - NHS Choices [Internet]. 2017 [cited 2017 Oct 10]. Available from: http://www.nhs.uk/Conditions/Typhoidfever/Pages/Prevention.aspx

26. Vaccine Information Statement | Typhoid | VIS | CDC [Internet]. [cited 2017 Oct 10]. Available from: https://www.cdc.gov/vaccines/hcp/vis/visstatements/typhoid.html

27. Waddington CS, Darton TC, Woodward WE, Angus B, Levine MM, Pollard AJ. Advancing the management and control of typhoid fever: a review of the historical role of human challenge studies. J Inf Secur. 2014;68(5):405-18. 
28. Caraway CT, Bruce JM. Typhoid fever epidemic following a wedding reception. Public Health Rep. 1961;76(5):427-30.

29. Michel R, Garnotel E, Spiegel A, Morillon M, Saliou P, Boutin J-P. Outbreak of typhoid fever in vaccinated members of the French armed forces in the Ivory Coast. Eur J Epidemiol. 2005;20(7):635-42.

30. Bajracharya D, Khan MI, lii AP, Shrestha P, Joshi N, Upreti SR, et al. 25 years after vi typhoid vaccine efficacy study, typhoid affects significant number of population in Nepal. PLoS One. 2014;9(1):e77974.

31. Bayram Y, Güdücüoğlu H, Otlu B, Aypak C, Gürsoy NC, Uluç H, et al. Epidemiological characteristics and molecular typing of Salmonella enterica serovar Typhi during a waterborne outbreak in eastern Anatolia. Ann Trop Med Parasitol. 2011;105(5):359-65

32. Centres for Disease Control and Prevention (CDC). Notes from the field. Salmonella Typhi infections associated with contaminated water-Zimbabwe, October 2011-may 2012. MMWR Morb Mortal Wkly Rep. 2012; 61(23):435.

33. Egoz N, Shihab S, Leitner L, Lucian M. An outbreak of typhoid fever due to contamination of the municipal water supply in northern Israel. Isr J Med Sci. 1988;24(11):640-3.

34. Roy JS, Saikia L, Medhi M, Tassa D. Epidemiological investigation of an outbreak of typhoid fever in Jorhat town of Assam. India Indian J Med Res. 2016;144(4):592-6.

35. Loharikar A, Newton A, Rowley P, Wheeler C, Bruno T, Barillas H, et al. Typhoid fever outbreak associated with frozen mamey pulp imported from Guatemala to the western United States, 2010. Clin Infect Dis Off Publ Infect Dis Soc Am. 2012;55(1):61-6.

36. Taylor A, Santiago A, Gonzalez-Cortes A, Gangarosa EJ. Outbreak of typhoid fever in Trinidad in 1971 traced to a commercial ice cream product. Am J Epidemiol. 1974;100(2):150-7.

37. al-Zubaidy AA, el Bushra HE, Mawlawi MY. An outbreak of typhoid fever among children who attended a potluck dinner at Al-Mudhnab, Saudi Arabia. East Afr Med J. 1995;72(6):373-5.

38. Ash I, Mckendrick GD, Robertson MH, Hughes HL. Outbreak of typhoid fever connected with corned beef. Br Med J. 1964;1 (5396):1474-8.

39. Hornick RB, Greisman SE, Woodward TE, DuPont HL, Dawkins AT, Snyder MJ. Typhoid fever: pathogenesis and immunologic control. N Engl J Med. 1970; 283(13):686-91.

40. World Health Organization. Foodborne disease outbreaks: Guidelines for investigation and control [Internet]. 2008 [cited 2016 Nov 17]. Available from: http://www.who.int/foodsafety/publications/foodborne_disease/ outbreak_guidelines.pdf

41. Pradier C, Keita-Perse O, Bernard E, Gisbert C, Vezolles MJ, Armengaud A, et al. Outbreak of typhoid fever on the French Riviera. Eur J Clin Microbiol Infect Dis Off Publ Eur Soc Clin Microbiol. 2000;19(6):464-7.

42. Anita S, Amir KM, Fadzilah K, Ahamad J, Noorhaida U, Marina K, et al. Risk factors for typhoid outbreak in Sungai Congkak Recreational Park, Selangor 2009. Med J Malaysia. 2012;67(1):12-6.

43. Coté TR, Convery $H$, Robinson D, Ries $A$, Barrett $T$, Frank $L$, et al. Typhoid fever in the park: epidemiology of an outbreak at a cultural interface. J Community Health. 1995;20(6):451-8.

Ready to submit your research? Choose BMC and benefit from:

- fast, convenient online submission

- thorough peer review by experienced researchers in your field

- rapid publication on acceptance

- support for research data, including large and complex data types

- gold Open Access which fosters wider collaboration and increased citations

- maximum visibility for your research: over $100 \mathrm{M}$ website views per year

At BMC, research is always in progress.

Learn more biomedcentral.com/submissions 